\title{
Comparison of Thermal Performance Equations in Describing Temperature- Dependent Developmental Rates of Insects: (III) Phenological Applications
}

\section{Authors: Pei-Jian Shi, Mei-Ling Fan, and Gadi V. P. Reddy}

This is a pre-copyedited, author-produced PDF of an article accepted for publication in Annals of the Entomological Society of America following peer review. The version of record, see complete citation below, is available online at: https://dx.doi.org/10.1093/aesa/sax063.

Shi, Pei-Jian, Mei-Ling Fan, and Gadi V. P. Reddy. "Comparison of Thermal Performance Equations in Describing Temperature-Dependent Developmental Rates of Insects: (III) Phenological Applications." Annals of the Entomological Society of America 110, no. 6 (November 2017): 558-564. DOI: 10.1093/aesa/sax063.

Made available through Montana State University's $\underline{\text { ScholarWorks }}$ scholarworks.montana.edu 


\title{
Comparison of Thermal Performance Equations in Describing Temperature-Dependent Developmental Rates of Insects: (III) Phenological Applications
}

\author{
Pei-Jian Shi, ${ }^{1,4}$ Mei-Ling Fan, ${ }^{1,4}$ and Gadi V. P. Reddy ${ }^{2,3}$ \\ ${ }^{1}$ Co-Innovation Centre for Sustainable Forestry in Southern China, Bamboo Research Institute, Nanjing Forestry University, 159 \\ Longpan Road, Nanjing 210037, China, ${ }^{2}$ Western Triangle Agricultural Research Center, Montana State University, 9546 Old Shelby \\ Road, P.O. Box 656, Conrad, MT 564925, ${ }^{3}$ Corresponding author, e-mail: reddy@montana.edu, and ${ }^{4}$ These two authors contributed \\ equally to this work
}

\begin{abstract}
The developmental times of poikilotherms at different stages are significantly affected by temperature. Most mathematical models describing the temperature-dependent developmental rates of poikilotherms are built according to the experimental data at various constant temperatures. However, these models can also be applied to the developmental rates at variable temperatures. It is more meaningful to use models to predict the occurrence times of pest insects that actually represent the completion for a particular developmental stage (e.g., hatching, pupation, eclosion) under a natural thermal environment. For some developmental stages, insects might experience a period of high temperatures. In this case, skewed bell-shaped nonlinear models are more reasonable than the linear and exponential models because in the high-temperature region the developmental rate decreases with temperature increasing. We used the accumulated developmental progress method that combines three representative nonlinear models to compare the model validity in predicting the egg's earliest hatching date of bamboo locust in different years. We found that for the springtime phenological event the simple Arrhenius' equation obtains the best goodness of fit. This study also provides a general $\mathrm{R}$ function that permits users to employ nonlinear parametric models to predict the occurrence times of insect phenology. In fact, if the investigation data cannot reflect the temperature-based phenological models proposed here, we have to consider whether the data set is reliable or whether the temperature is the crucial factor that determines the occurrence time of interest. The present study is valuable for the integrated management of pest insects because the biological or chemical control timing relies on the prediction on the occurrence time of phenological events.
\end{abstract}

In the investigation of temperature-dependent developmental rates of ectotherms and plants, mathematical models are largely used (Shi et al. 2016, 2017c; Ratkowsky and Reddy 2017). These models are usually built based on the experimental observations of developmental rates at constant temperatures (that are conducted in incubators and greenhouses), conclusions drawn from which are criticized for not being applied to the actual occurrences in a variable natural thermal environment (Ring and Harris 1983, Stevenson et al. 2008). However, models can actually be used to predict the emergence of insects on natural conditions just accumulating the developmental rates to one (Wagner et al. 1984). The developmental rate represents the developmental progress of a specific stage at a unit time (i.e., per hour, per day), so $100 \%$ represents the developmental completion of this stage. This method was proposed in the past; however, it has been seriously overlooked and only few studies have employed this method (e.g., Wagner et al. 1984; Sugiura et al. 1991; Ungerer et al. 1999; Shi et al. 2017a). We referred to this approach as the accumulated developmental progress (ADP) method. The ADP method can combine various temperature-dependent developmental rate models. Recently, Shi et al. (2017a) compared the degree-days method (Ring and Harris 1983), the number of days transformed to the standard temperature (DTS) method (Aono 1993), and the ADP method that uses the Arrhenius' equation. They also found that the ADP method could yield the least prediction error for the first flowering date of Japanese cherry trees (Prunus yedoensis Matsum [Rosales: Rosaceae]). However, in Shi et al. (2017a), the procedure of the ADP approach is not general, which is only applicable to the Arrhenius' equation. In fact, the Arrhenius' equation is effective only for the low- and mid-temperature regions. The developmental rate curve of ectotherms has different track tendencies in the low-, mid-, and 
high-temperature regions (Campbell et al. 1974; Shi et al. 2017c). In the mid-temperature regions, a linear relationship between temperature and developmental rate exists, whereas in the high-temperature region (beyond a critical point of temperature), the developmental rate will decrease with temperature increasing (Uvarov 1931; Campbell et al. 1974; Wagner et al. 1984; Ratkowsky and Reddy 2017; Shi et al. 2017c). Figure 1 exhibits the temperature-dependent developmental rates of female pupae of cotton bollworm (Helicoverpa armigera Hübner [Lepidoptera: Noctuidae]; Wu et al. 2009) using a nonlinear model that will be introduced in the section of Materials and Methods.

Phenological events of insects are very important because the occurrence times of many agricultural and forest pest insects directly affect the output of crops and forest ecological security. The insects are good study objects for phenology and different generations and developmental stages are both largely affected by air temperatures at different seasons. Locusts are widely concerned because they can lead to huge damage to crops and forests. Locust plagues cannot be neglected today, which still might cause famines in several regions of the planet (Lima 2007). Under global climate change, the physiology, geographical distribution and phenology of many species especially for plants and poikilotherms are varying with changing climate (Hughes 2000). Some studies have shown that the outbreaks of locusts usually occurred in the historical 'cold' periods (Stige et al. 2007; Tian et al. 2011). Yu et al. (2013) compared the population dynamics of locust swarms on the plains of North America and East Asia. The Rocky Mountain locust (Melanoplus spretus Walsh [Orthoptera: Acrididae]) broke out in a large number in the period 1874-1878 AD, but then slowly disappeared. The wide use of chemical pesticides was considered a critical factor leading to the disappearance in the locust swarm. However, Yu et al. (2013) debated on this explanation by using the historical outbreaks of Locusta migratoria manilensis Meyen (Orthoptera: Acrididae) in China. Although the chemical pesticides were widely used in China in the 1950s, the locust swarms frequently occurred in the 1960s. They analyzed the

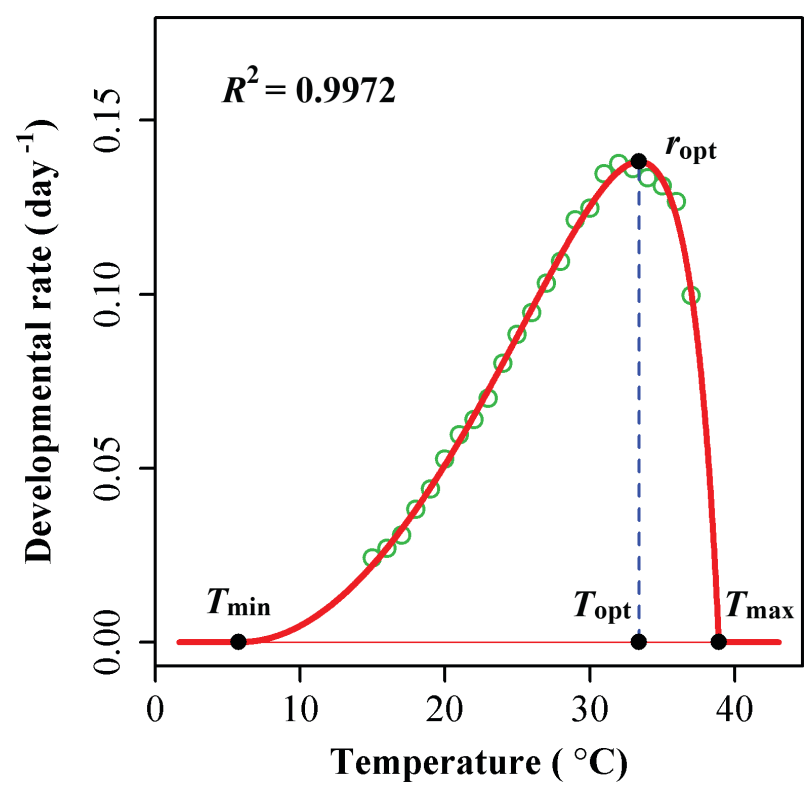

Fig. 1. Nonlinear temperature effect on the female pupal developmental rate of cotton bollworm. The data represented by open circles came from Wu et al. (2009); the LRF equation (represented by the curve; see the section of Materials and Methods for detail) was used to fit these data. The four parameters in the LRF equation are all marked on the curve. climate extremes and monthly temperature-precipitation combinations in two regions, and found that the differences in the frequencies of these climate combinations resulted in the contrasting locust fates in the two regions. We need to point out that during 1959-1961 there were at least 40 million farmers died from the Great Famine in Mainland China (Jin 1993) and the farming management and pest control were slack in the subsequent years, which might be a factor resulting in the locust swarms of the 1960s. However, climate especially temperature and drought are two crucial climatic deriving factors for the outbreaks of locusts. In southern China, bamboos especially moso bamboo, are important components of forest landscapes. Bamboo shoots can also be used as traditional foods in China. Ceracris kiangsu Tsai (Orthoptera: Acrididae) is a serious pest insect of bamboos (Cheng et al. 2010). It mainly feeds on bamboo leaves and can damage crops (such as rice and wheat) if bamboos are unavailable. The overwintering bamboo locust eggs usually hatch in mid spring in southern China.

In this study, we developed a general procedure of the ADP method that can combine any parametric models. Then using the time series of egg's hatching time of bamboo locusts we compared three nonlinear models combined in the ADP method. In addition, we also compared the ADP method with the accumulated degreedays (ADD) and DTS methods (Shi et al. 2017a) to check which method is the best for describing the influence of temperature on the occurrence time of the pest insect. The drawn conclusion can also be extended to others.

\section{Materials and Methods}

\section{Data}

The data came from Huang et al. (1998) that report the yearly occurrence times from 1987 to 1997 of bamboo locusts in Guangning County, Guangdong Province. Each year eight to ten sample sites $(0.1$ $\mathrm{hm}^{2}$ each) along mountain slopes where the eggs had been observed and collected. The first hatching time each year was recorded as the earliest hatching time.

The climatic data of Guangning County from 1986 to 1997 are obtained from China Meteorological Administration (http://data. cma.cn/).

\section{Methods}

\section{ADD Method}

Assume that the requirement of heat sum for completing a specific developmental stage is a constant $(=k)$. There are two important parameters in the ADD method: the starting date $(S)$ and the base temperature $\left(T_{0}\right)$. The starting date is the time when heat accumulation starts that is assumed to be the same for each year; the base temperature, which is also assumed to be a constant, is the threshold beyond which temperatures can affect the development process. Then

$$
k_{i}=\sum_{j=S}^{E_{i}}\left(T_{i j}-T_{0}\right)
$$

Here, $E_{i}$ represents ending date when the phenological event of interest occurs in the $i$-th year; the subscript $j$ represents the $j$-th day; $T_{i j}$ represents the daily mean air temperature on the $j$-th day in the $i$-th year. Because the ending dates and daily mean air temperatures in one year are different from those in another year, there are differences in calculated heat sums among different years. The mean heat sum of time series is regarded as the expected (or called 'critical') value to predict the occurrence dates in various years. The day when 
temperature accumulation reaches or exceeds the expected value of the required heat sum is regarded as the predicted occurrence date. For different combinations of a vector of starting dates and a vector of base temperatures, the final estimates on starting date and base temperature are determined if the calculated root mean square errors (RMSEs) is the least. However, in practice, the RMSE usually is a monotonous function of starting date or base temperature for some data sets. Thus, a feasible approach is to first determine the starting date and then to determine another parameter. The occurrence date (unit: day of a year; DOY) is considered to be negatively related to the average of daily mean air temperatures during the period from the starting date to the ending date (Aono 1993; Shi et al. 2017a). Therefore, we can determine starting date based on the least negative correlation coefficient. We can further determine the base temperature that can result in the least RMSE from a vector of candidate base temperatures.

\section{DTS Method}

This method is based on the Arrhenius's equation (Arrhenius 1889):

$$
r=\exp \left(B-\frac{E_{a}}{R T}\right)
$$

Here, $r$ represents developmental rate at absolute temperature $T$ in Kelvin; $B$ and $E_{a}$ are constants; $R$ is the universal gas constant (=1.987 $\mathrm{cal} \mathrm{mol}^{-1} \mathrm{~K}^{-1}$ ). The multiplication between developmental rate and developmental time is a constant $(=1)$ because the definition of developmental rate is the reciprocal of developmental time. Then there is:

$$
r_{1} t_{1}=r_{2} t_{2}=\ldots=r_{s} t_{s}=1
$$

Here, $r_{s}$ and $t_{s}$ represent the developmental rate and developmental time at a referred 'standard' temperature $T_{s}(=298.15 \mathrm{~K})$. The subscripts 1 and 2 represent the first and second day, etc. Thus the following equation can be obtained

$$
t_{s}=t_{i j} \frac{r_{i j}}{r_{s}}=t_{i j} \frac{\exp \left[B-E_{a} /\left(R T_{i j}\right)\right]}{\exp \left[B-E_{a} /\left(R T_{s}\right)\right]}=t_{i j} \exp \left[\frac{E_{a}\left(T_{i j}-T_{s}\right)}{R T_{i j} T_{s}}\right]
$$

Then we can calculate the number of days transferred to the standard temperature for the $j$-th day (with daily mean air temperature $=T_{i j}$ ) of the $i$-th year as follows:

$$
D T S_{i j}=\exp \left[\frac{E_{a}\left(T_{i j}-T_{s}\right)}{R T_{i j} T_{s}}\right]
$$

Note that the developmental time $t_{i j}$ in equation 5 is changed to be 1 because the corresponding time scale is only $1 \mathrm{~d}$ on the $j$-th day. The daily $D T S_{i j}$ actually represents the number of days at a standard temperature transferred from $1 \mathrm{~d}$ at an observed daily mean air temperature. The daily $D T S_{i j}$ also represents the standardized developmental rate at temperature $T_{i j}$ relative to the developmental rate at the standard temperature $T_{s}$.

For $1 \mathrm{yr}$, we can calculate the accumulation of daily DTS values from the starting date to the occurrence date (i.e., the ending date). Then the yearly mean of the accumulations of daily DTS values for different years can be regarded as the critical accumulation of daily
DTS values. Similar to the ADD method, the occurrence dates for various years can be predicted when the calculated accumulation of daily DTS values equals or exceeds the yearly mean of the accumulations of daily DTS values. The least RMSE will determine the starting date and the parameter $E_{a}$.

\section{ADP Method}

The principle of the ADP method is easy to understand. The developmental rate is defined as the progress of completing the growth development in a unit time. Thus, the occurrence date can be predicted when the accumulated developmental rates equals or exceeds 100\% (Wagner et al. 1984; Sugiura et al. 1991; Ungerer et al. 1999; Shi et al. 2017a). In the ADP method, besides the Arrhenius' equation, the developmental rate can be any other nonlinear parametric models (Ratkowsky and Reddy 2017). In this article, we mainly considered three nonlinear models: the Arrhenius' equation, Logan equation, and Lobry-Rosso-Flandrois (LRF) equation.

Arrhenius' equation

$$
r=\exp \left(B-\frac{E_{a}}{R T}\right) \times 10^{12}
$$

Here, the factor $10^{12}$ is used to reduce the size of parameter $B$. The unit of $E_{a}$ is chosen as $\mathrm{kcal} \cdot \mathrm{mol}^{-1}$. Note that we need not multiply equation 2 by such a factor in the DTS method because it cancels out in equation 4 by the division.

Logan equation (Logan et al. 1976, Shi et al. 2011):

$$
r=\psi\left[\exp (\rho T)-\exp \left(\rho T_{\max }-\frac{T_{\max }-T}{z}\right)\right]
$$

Here, $r$ represents developmental rate at temperature $T$ in ${ }^{\circ} \mathrm{C} ; T_{\text {max }}$ represents the 'notional' upper developmental threshold; $\Psi, \rho$, and $z$ are constants. This equation has four parameters to be fitted.

(3) LRF equation (Lobry et al. 1991; Rosso et al. 1993):

$$
r=\frac{r_{\mathrm{opt}}\left(T-T_{\max }\right)\left(T-T_{\min }\right)^{2}}{\left(T_{\mathrm{opt}}-T_{\min }\right)\left[\left(T_{\mathrm{opt}}-T_{\min }\right)\left(T-T_{\mathrm{opt}}\right)-\left(T_{\mathrm{opt}}-T_{\max }\right)\left(T_{\mathrm{opt}}+T_{\min }-2 T\right)\right]}
$$

when $T_{\min } \leq T \leq T_{\max } ; r=0$, when $T \notin\left(T_{\min }, T_{\max }\right)$. See Fig. 1 for intuitively understanding the meanings of four parameters in the LRF equation. There are other models that have four parameters including the notional lower and upper developmental thresholds (Shi et al. 2016, 2017b; Ratkowsky and Reddy 2017). However, the LRF equation is demonstrated to be the best from the trade-off between the goodness of fit and the parameter performance of nonlinearity (Ratkowsky and Reddy 2017). The Logan equation doesn't have the lower developmental threshold $\left(T_{\min }\right)$ that might limit the fitting flexibility at low temperatures. Thus, the Logan equation might be more suitable for describing the effect of low temperature in spring than the LRF equation.

\section{Influence of Winter LowTemperatures Before the Starting Date on the Occurrence Date}

To examine whether the occurrence date (i.e., the hatching time) is significantly affected by winter low temperatures before the starting date, the mean daily minimum temperature from 1 November 
of the previous year to the starting date $\left(x_{1}\right)$, and the number of days with daily minimum temperature $\leq$ a critical low temperature $\left(x_{2}\right)$ were used to explain the prediction residuals using the method that can obtain the least RMSE among the ADD, DTS and ADP methods. The semi-parameter generalized additive model (Hastie and Tibshirani 1990; Wood 2006) was used to analyze the above relationship:

$$
\mathrm{E}\left(y \mid x_{1}, x_{2}\right)=\alpha+s\left(x_{1}\right)+\beta x_{2}
$$

Here, $y$ represents the prediction residuals; $s(\cdot)$ represents a unspecified smooth ('nonparametric') function; $\alpha$ and $\beta$ are constants.

The analysis is divided to two steps: (1) to a specific phenological method to find a starting date, check the effect of the daily mean temperatures from the starting date to the hatching date on the hatching time (in DOY), and provide the initial predicted occurrence time; 2) to use the prediction residuals from the first step as the response variable, and examine the effects of the mean of daily minimum temperatures (from 1 November of the previous year to the hatching date) and the duration of low temperatures (represented by the number of days with daily minimum temperature $\leq$ a critical low temperature) on the prediction residuals. Consequently, the total prediction errors can be less than for using only the first step.

The $\mathrm{R}$ functions for implementing the ADD and DTS methods in Shi et al. (2017a) were used. We developed a general ADP method and the relevant $\mathrm{R}$ function that can combine any nonlinear parametric models (Supp Material 1 [online only]). The statistical software R (version 3.2.2; R Core Team 2015) was used to carry out the calculations and to make the plots.

\section{Results}

Table 1 shows the parameter estimates and goodness of fits (represented by RMSE) of the five methods. The ADP method with the Arrhenius' equation produces the least RMSE. The ADP method with the Logan equation can also obtain a less RMSE, but slightly greater than that from the Arrhenius' equation. Figure 2 shows the predicted ADPs in different years and the daily mean air temperatures from the starting date (the 36th DOY) to the observed hatching date. Because the ADP method with the Arrhenius' equation obtains the least RMSE, the prediction residuals were further analyzed using winter low temperatures. We found that the explainable deviance is maximum $(=41.7 \%)$ when the critical low temperature is equal to $-0.91^{\circ} \mathrm{C}$. That means, the mean daily minimum air temperatures from the 1 November to the starting date (i.e., 5 February) and the number of days with daily minimum temperature $\leq-0.91^{\circ} \mathrm{C}$ have explained $41.7 \%$ of residuals that cannot be explained by using the ADP method. After considering the effects of winter low temperatures and lasting time, the prediction errors are further decreased. After considering the effects of winter low temperatures and the lasting time, the RMSE is decreased to $3.2 \mathrm{~d}$, which can fully explain deviance reaching to $86.2 \%$.

\section{Discussion}

In our recent study on the first flowering date of cherry trees in early spring, the ADP approach with the Arrhenius' equation has demonstrated to be better than the ADD and DTS methods (Shi et al. 2017a). However, we did not compare whether the ADP method with the Arrhenius' equation is also better than the ADP methods with other nonlinear models for spring phenology. In the present study, the ADP method with the Arrhenius' equation is demonstrated to be superior to the others. In the low-temperature region that the eggs of bamboo locusts have experienced, the developmental rate theoretically grows exponentially with increasing temperature. The Logan and Arrhenius' equations have better flexibility in data fitting than the LRF equation because the curvature of the LRF equation used is limited relative to the five-parameter LRF equation (Shi et al. 2017b) in describing the effect of low temperature:

$$
r=r_{\mathrm{opt}}\left\{\frac{\left(T-T_{\max }\right)\left(T-T_{\min }\right)^{2}}{\left(T_{\mathrm{opt}}-T_{\min }\right)\left[\left(T_{\mathrm{opt}}-T_{\min }\right)\left(T-T_{\mathrm{opt}}\right)-\left(T_{\mathrm{opt}}-T_{\max }\right)\left(T_{\mathrm{opt}}+T_{\min }-2 T\right)\right]}\right\}^{\delta}
$$

There is an additional exponent $\delta$ that can make the curve fitting more flexible. However, too many parameters will increase the complexity of a model and usually destroys the good estimation properties that a simpler model without the exponent might have (Ratkowsky 1983; Shi et al. 2017b). Although the LRF equation has a good property in parameter performance of nonlinearity, it cannot accurately describe the effect of low temperature on developmental rate for phenological data. The Logan equation exhibits an approximation to the exponential equation in the low-temperature region. If there were phenological dates occurring in summer, the Logan and LRF equations should be more suitable than the Arrhenius' equation

\begin{tabular}{|c|c|c|c|}
\hline Method & Estimate of the starting date (DOY) & Estimate(s) on the model parameter(s) & RMSE (d) \\
\hline $\mathrm{ADD}$ & 37 & $\widehat{T}_{0}=11.9\left({ }^{\circ} \mathrm{C}\right)$ & 4.5741 \\
\hline DTS & 36 & $\widehat{E}_{a}=27.34\left(\mathrm{kcal} \mathrm{mol}^{-1}\right)$ & 4.2218 \\
\hline ADP-Arrhenius & 36 & $\begin{array}{l}\hat{E}_{a}=27.72\left(\mathrm{kcal} \mathrm{mol}^{-1}\right) \\
\hat{B}=16.04 \text { (unitless) }\end{array}$ & 4.1829 \\
\hline ADP-Logan & 36 & $\begin{array}{l}\hat{\psi}=7.3749 \times 10^{-4} \text { (unitless) } \\
\hat{\rho}=0.1646 \text { (unitless) } \\
\hat{T}_{\max }=48.96\left({ }^{\circ} \mathrm{C}\right) \\
\hat{z}=2.5861 \text { (unitless) }\end{array}$ & 4.1839 \\
\hline ADP-LRF & 35 & $\begin{array}{l}\hat{r}_{\text {opt }}=0.0653\left(\mathrm{day}^{-1}\right) \\
\hat{T}_{\text {opt }}=32.72\left({ }^{\circ} \mathrm{C}\right) \\
\hat{T}_{\min }=3.79\left({ }^{\circ} \mathrm{C}\right) \\
\hat{T}_{\max }=32.89\left({ }^{\circ} \mathrm{C}\right)\end{array}$ & 4.3086 \\
\hline
\end{tabular}

Table 1. Comparison of parameter estimates of the five methods for bamboo locust hatching time 

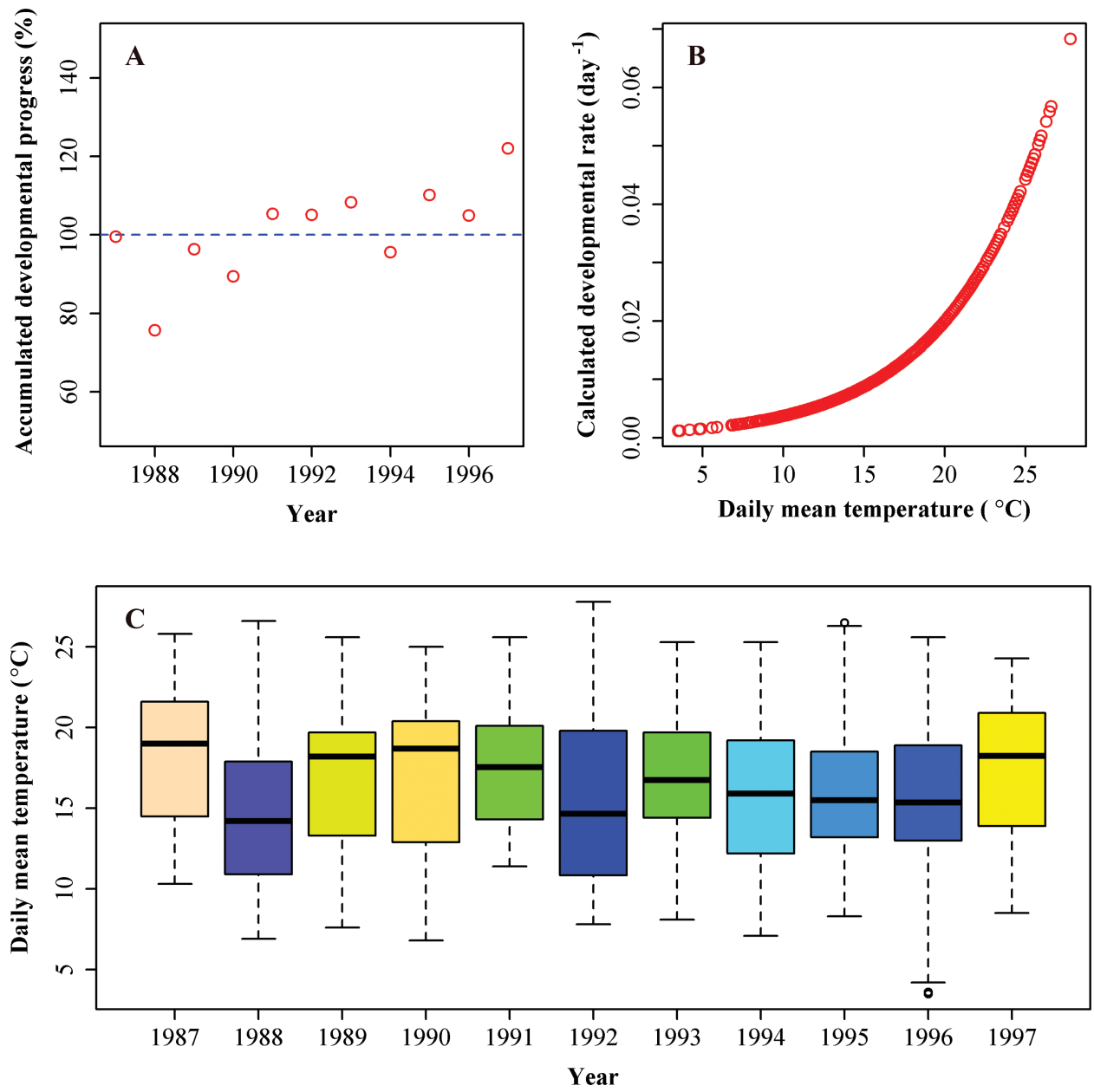

Fig. 2. Fitted results using the ADP method with the Arrhenius' equation. (A) Predicted annual ADP; (B) Predicted developmental rate curve; (C) Daily mean air temperatures from the starting date (i.e., $36 \mathrm{DOY}$ ) to the observed hatching dates for various years.

considering that the latter cannot be used to describe the developmental rates in the high-temperature region.

The starting dates predicted by the five methods appear to be approximate. However, the ADD method yields the greatest prediction error. Although there are differences among several methods, the predicted occurrence dates are also approximate. The predicted biological zero (namely the lower developmental threshold) from the ADD method is $11.9^{\circ} \mathrm{C}$ for the egg development, which is in accord with the previous reports in other locusts ranging from 10 to $12^{\circ} \mathrm{C}$ (Hao and Kang 2004; Chen et al. 2009). It is easy to explain why the ADD method can also obtain a good prediction. Because the most daily mean temperatures from the starting date to the hatching date are large than $12^{\circ} \mathrm{C}$ (Fig. 2), the developmental rate curve approximates to a straight line in the mid-temperature region (Campbell et al. 1974). Although the Arrhenius, Logan and LRF equations are all nonlinear, they all can be approximated to the straight line between 12 and $25^{\circ} \mathrm{C}$. Of course, there are also some days with mean temperature lower than $12^{\circ} \mathrm{C}$ especially for the predicted curve by the LRF equation, which renders the ADP method with the Arrhenius' equation to be the best.

The present study confirms the important influence of winter low temperatures on the occurrence time of insects in spring. In fact, winter low temperatures can significantly affect the survival of overwintering insects, which further determines the northern distribution in the Northern Hemisphere and population density of the following year generation (Uvarov 1931; Ungerer et al. 1999; Friedenberg et al. 2008; Shi et al. 2012; Valtonen et al. 2014). We did not use the spline function in the generalized additive model to examine the effect of the second predictor, the number of days with low temperature $\leq-0.9^{\circ} \mathrm{C}$ (equation 9), because the sample size is not large, only having 11-yr historical records. However, the linear approximation for the second predictor has been demonstrated to be feasible, the two predictors explain $42 \%$ deviance of the prediction residuals from the ADP method with the Arrhenius' equation. When the second predictor was dropped, the explainable deviance is decreased to $25.5 \%$ only. Thus, the absolute quantity of winter low temperatures and duration of extreme low temperatures are two crucial factors that affect the hatching time. Interestingly, it is well known that there are two stages of dormancy states for plants: endodormancy and ecodormancy. For completing the first stage, only chilling requirement must be met; for completing the second stage, forcing requirement (i.e., heat sum or rate sum) must be met. And spring warming only speeds the second stage. We believe that there are two corresponding stages for overwintering insects: diapause and ecodormancy. In entomology, there is no terms of endodormancy. Thus, 'endodormancy' actually has been substituted by 'diapause'. In the present study, we demonstrate that winter low temperatures are important to break the state of winter diapause for insects. And springtime temperatures are important to break the state of ecodormancy in spring. 

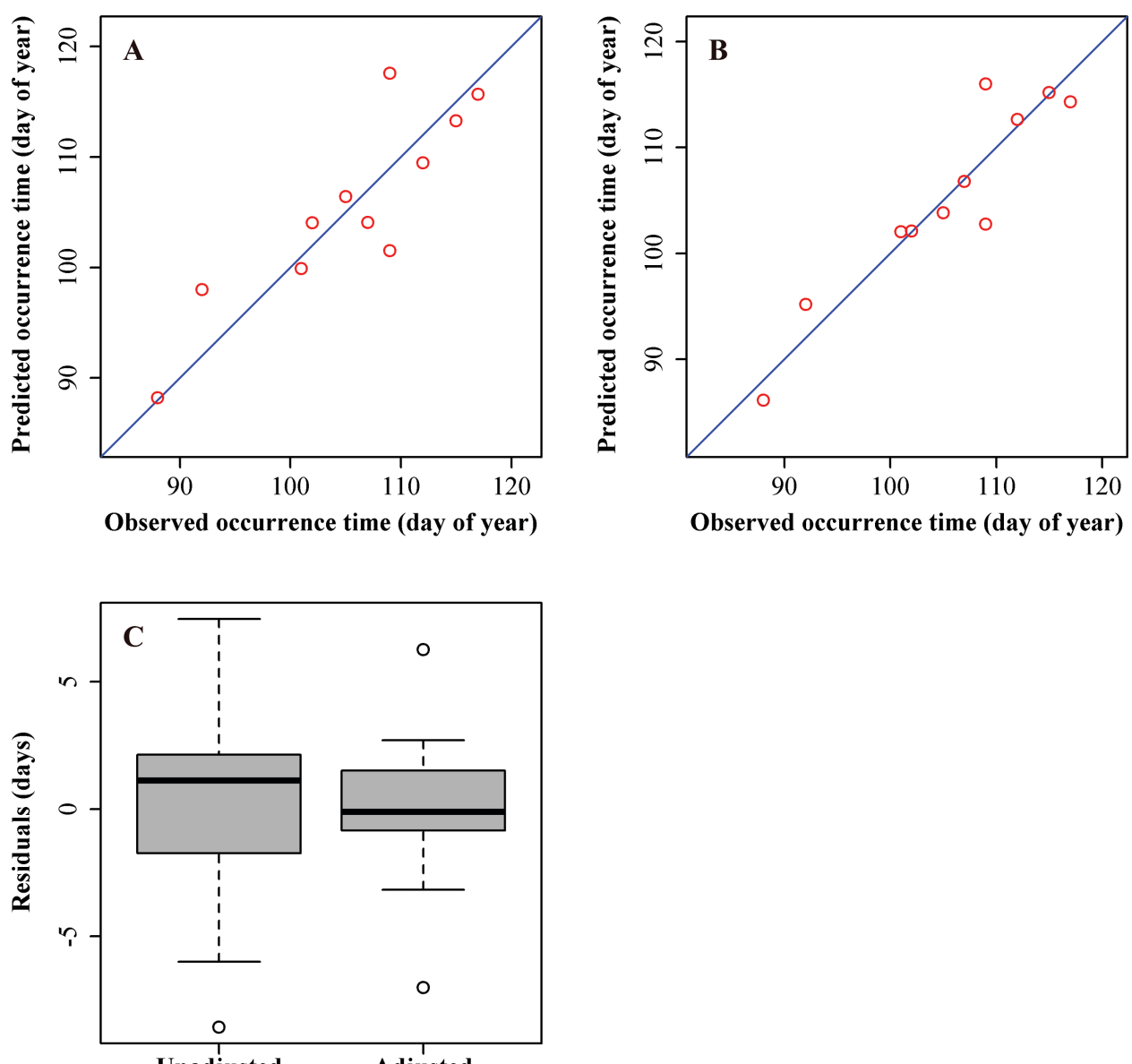

Unadjusted Adjusted

Fig. 3. Comparison of the two predictions: (A) without considering the effects of winter low temperatures and duration of extreme low temperatures, and (B) with considering the effects of winter low temperatures and duration of extreme low temperatures. (C) Boxplots for the prediction residuals between two cases for different years.

In our recent study on the first flowering date of Japanese cherry trees in the Wuhan University campus, 67-yr time series was used based on the ADP method with the Arrhenius' equation (Shi et al. 2017a). To observe the spring flowering date of plants in the campus is apparently easier to deal than to observe the hatching time of bamboo locust's eggs in field. For the former, the trees are planted along a road in the campus (which means that these trees are keeping high temporal synchronicity and close spatial proximity for the first flowering time), and flowering events can be directly observed by eyes regardless of the effects of spatial heterogeneity and microenvironmental factors. Thus, the long-term records can be easily obtained. However, for the earliest hatching time of bamboo locust's eggs, the situation is rather different from the first flowering time of Japanese cherry trees. The influence of spatial heterogeneity and micro-environmental factors must be considered. In this case, different sites in mountain slopes must be chosen to reduce the effects of the two types of factors. Thus, 8-10 sampling sites were used per year to determine the hatching time. Then, without any funding supporting, the field experiment would not be continuously conducted. Unluckily, the experiment of bamboo locusts was stopped after 1997 because the project had been completed. Consequently, there were no further funds to continue that experiment. Thus, the data set exists only from 1987 to 1997 . However, the data set is sufficient for us to exhibit the application of the ADP method and model comparison. The RMSE without considering the effects of winter low temperatures and duration of low temperatures appears to be large for 11-yr data set. However, there are two extreme prediction errors (and the residuals $>7 \mathrm{~d}$ ) in 1988 and 1997. The median of daily mean air temperatures are the lowest and highest for these years, respectively (Fig. 2C). Too low of the daily mean air temperatures leads the predicted ADP to be lower than $100 \%$; and conversely, too high of a daily mean air temperature leads that to be higher than $100 \%$ (Fig. 2A). Even considering the effects of winter low temperatures, the prediction errors are still extreme (Fig. 3C). We need to point out that the climatic site where the climate data are available is actually far from the experimental site. We hypothesize that the temperatures at experimental site might exhibit less extreme tendencies in these $2 \mathrm{yr}$; otherwise, the observations at the experimental site possess greater errors. Anyhow, the reason of leading to significant prediction errors should not be attributed to the method used.

\section{Supplementary Material}

Supplementary material is available at Annals of the Entomological Society of America online.

\section{Acknowledgments}

We are deeply thankful to Prof. Hsin-i Wu (Texas A \& M University, College Station) for commenting on the early version of this manuscript. We also thank Dr. Carlos Blanco and two reviewers for providing valuable comments on this manuscript. P.-J.S. was supported by the National Natural Science 
Foundation of China (31400348), Jiangsu Provincial Forestry Innovation Project (LYSX[2016]04), and the PAPD of Jiangsu Province. This material is also partly based upon work that is supported to G.V.P.R. by the National Institute of Food and Agriculture, U.S. Department of Agriculture, Hatch project under Accession (1009746).

\section{References Cited}

Aono, Y. 1993. Climatological studies on blooming of cherry tree (Prunus yedoensis) by means of DTS method. Bull. Univ. Osaka Pref., Ser. B 45: 155-192. (in Japanese with English abstract)

Arrhenius, S. A. 1889. Über die Dissociationswärme und den Einfluss der Temperatur auf den Dissociationsgrad der Elektrolyte. Z. Phys. Chem. 4 96-116.

Campbell, A., B. D. Frazer, N. Gilbert, A. P. Gutierrez, and M. Mackauer. 1974. Temperature requirements of some aphids and their parasites. J. Appl. Ecol. 11: 431-438.

Chen, G.-P., S.-G. Hao, B.-P. Pang, and L. Kang. 2009. Effect of photoperiod on the development, survival, eclosion and reproduction of 4th instar nymph of three grasshopper species in Inner Mongolia. Chin. Bull. Entomol. 46(1): 51-56. (in Chinese with English abstract)

Cheng, J., Y. Pan, and J. Shu. 2010. The research and development of the Ceracris kiangsu Tsai. Shandong For. Sci. Tech. [No Vol.](2): 116-119. (in Chinese with English abstract)

Friedenberg, N. A., S. Sarkar, N. Kouchoukos, R. F. Billings, and M. P. Ayres. 2008. Temperature extremes, density dependence, and southern pine beetle (Coleoptera: Curculionidae) population dynamics in east Texas. Environ. Entomol. 37: 650-659.

Jin, H. 1993. The memo of Three Years of Natural Disasters. Chin. J. Appl. Sociol. 13(z2): 13-23. (in Chinese)

Hao, S.-G., and L. Kang. 2004. Effects of temperature on the post-diapause embryonic development and the hatching time in three grasshopper species (Orth., Acrididae). J. Appl. Ent. 128: 95-101.

Hastie, T., and R. Tibshirani. 1990. Generalized additive models. Chapman and Hall, London, UK.

Huang, H., L. Zheng, X. Huang, S. Huang, and G. Tong. 1998. Prediction of the incubation period of yellow-spined bamboo locust. Guangdong For. Sci. Tech. 14(2): 23-26. (in Chinese with English abstract)

Hughes, L. 2000. Biological consequences of global warming: is the signal already. Trend. Ecol. Evol. 15: 56-61.

Lima, M. 2007. Locust plagues, climate variation, and the rhythms of nature. Proc. Natl. Acad. Sci. USA 104: 15972-15973.

Lobry, J. R., L. Rosso, and J. P. Flandrois. 1991. A FORTRAN subroutine for the determination of parameter confidence limits in non-linear models. Binary 3: 86-93.

Logan, J. A., D. J. Wollkind, S. C. Hoyt, and L. K. Tanigoshi. 1976. An analytic model for description of temperature dependent rate phenomena in arthropods. Environ. Entomol. 5: 1133-1140.

R Core Team. 2015. R: a language and environment for statistical computing. R Foundation for Statistical Computing, Vienna, Austria. (https://www.Rproject.org/).

Ratkowsky, D. A. 1983. Nonlinear Regression Modeling: a unified practical approach. Marcel Dekker, New York, NY.

Ratkowsky, D. A., and G. V. P. Reddy. 2017. Empirical model with excellent statistical properties for describing temperature-dependent developmental rates of insects and mites. Ann. Entomol. Soc. Am., 110: 302-309.

Ring, D. R., and M. K. Harris. 1983. Predicting pecan nut casebearer (Lepidoptera: Pyralidae) activity at College Station, Texas. Environ. Entomol. 12: 482-486.
Rosso, L., J. R. Lobry, and J. P. Flandrois. 1993. An unexpected correlation between cardinal temperatures of microbial growth highlighted by a new model. J. Theor. Biol. 162: 447-463.

Shi, P., F. Ge, Y. Sun, and C. Chen. 2011. A simple mode for describing the effect of temperature on insect developmental rate. J. Asia-Pacific Entomol. 14: $15-20$.

Shi, P., G. V. P. Reddy, L. Chen, and F. Ge. 2016. Comparison of thermal performance equations in describing temperature-dependent developmental rates of insects: (I) empirical models. Ann. Entomol. Soc. Am. 109: 211-215.

Shi, P., Z. Chen, G. V. P. Reddy, C. Hui, J. Huang, and M. Xiao. 2017a. Timing of cherry tree blooming: contrasting effects of rising winter low temperatures and early spring temperatures. Agric. For. Meteorol. 240: 78-89.

Shi, P.-J., M.-L. Fan, D. A. Ratkowsky, J.-G. Huang, H.-I Wu, L. Chen, S.Y. Fang, and C.-X. Zhang. 2017b. Comparison of two ontogenetic growth equations for animals and plants. Ecol. Model. 349: 1-10.

Shi, P.-J., G. V. P. Reddy, L. Chen, and F. Ge. 2017c. Comparison of thermal performance equations in describing temperature-dependent developmental rates of insects: (II) two thermodynamic models. Ann. Entomol. Soc. Am. 110: 113-120.

Shi, P., B. Wang, M. P. Ayres, F. Ge, L. Zhong, and B.-L. Li. 2012. Influence of temperature on the northern distribution limits of Scirpophaga incertulas Walker (Lepidoptera: Pyralidae) in China. J. Therm. Biol. 37: 130-137.

Stevenson, D. E., G. J. Michels, J. B. Bible, J. A. Jackman, and M. K. Harris. 2008. Physiological time model for predicting adult emergence of western corn rootworm (Coleoptera: Chrysomelidae) in the Texas High Plains. J. Econ. Entomol. 101: 1584-1593.

Stige, L. C., K.-S. Chan, Z. Zhang, D. Frank, and N. C. Stenseth. 2007. Thousand-year-long Chinese time series reveals climatic forcing of decadal locust dynamics. Proc. Natl. Acad. Sci. USA 104: 16188-16193.

Sugiura, T., S. Ono, and F. Kamota. 1991. A model for developmental rate from rest break of flowering of Japanese pear. J. Agric. Meteorol. 46: 197203. (in Japanese with English abstract)

Tian, H., L. C. Stige, B. Cazelles, K. L. Kausrud, R. Svarverud, N. C. Stenseth, and Z. Zhang. 2011. Reconstruction of a 1,910-y-long locust series reveals consistent associations with climate fluctuations in China. Proc. Natl. Acad. Sci. USA 108: 14521-14526.

Ungerer, M. J., M. P. Ayres, and M. J. Lombardero. 1999. Climate and the northern distribution limits of Dendroctonus frontalis Zimmermann (Coleoptera: Scolytidae). J. Biogeogr. 26: 1133-1145.

Uvarov, B. P. 1931. Insects and climate. Trans. Entomol. Soc. London 79: $1-232$.

Valtonen, A., R. Leinonen, J. Pöyry, H. Roininen, J. Tuomela, and M. P. Ayres. 2014. Is climate warming more consequential towards poles? The phenology of Lepidoptera in Finland. Glob. Change Biol. 20: 16-27.

Wagner, T. L., H.-I Wu, P. J. H. Sharpe, R. M. Schoolfield, and R. N. Coulson. 1984. Modelling insect development rates: a literature review and application of a biophysical model. Ann. Entomol. Soc. Am. 77: 208-225.

Wood, S. N. 2006. Generalized additive models: an introduction with R. Chapman \& Hall/CRC, London, UK.

Wu, K.-J., P.-Y. Gong, and Y.-M. Ruan. 2009. Estimating developmental rates of Helicoverpa armigera (Lepidoptera: Noctuidae) pupae at constant and alternating temperatures by nonlinear models. Acta Entomol. Sin. 52: 640-650. (in Chinese with English abstract)

Yu, G., X. Ke, H. D. Shen, and Y. F. Li. 2013. An analysis of the contrasting fates of locust swarms on the plains of North America and East Asia. Biogeosciences 10: 1441-1449. 